\title{
Integrating diverse information resources: capturing meaning in a sea of data
}

\author{
Dr. Matthew Morgenstern \\ Xerox Design Research Institute \\ 502 Rhodes Hall, Cornell University, Ithaca, NY 14853 USA \\ Tel:(607)255-9899, Fax:(607)254-4742, Morgenstern@dri.cornell.edu
}

\begin{abstract}
The growth of networked databases and other multimedia resources heightens the need for effective means of integrating diverse information. To use multiple information sources effectively we need to understand the different meanings of data from different sources. Describing the semantics and context of the data makes it possible to bridge these differences. When we search indices we really are asking for data with a certain meaning. Yet our search terms often have different interpretations in different databases. As we rely increasingly on intelligent computational agents to filter the ever growing information overload, we must describe enough of the meaning to these agents so that they can manage the data effectively for our benefit.
\end{abstract}

\section{Keywords}

Metadata, heterogeneity, semantics, integration, intelligent systems, hyper-documents, information technology tools, databases, Web, HTML, SGML.

\section{INTRODUCTION}

The explosive growth of networked databases, structured information sources, and other multimedia data heightens the need to describe the meaning and context of the data so as to understand and utilize it effectively. We can use multiple information sources only if we understand what the data from each means: what are the differences in meaning and how the data may be translated to bridge these differences. How such meaning is described and communicated to us, and to our systems, is worthy of significant attention.

We recognized information overload even before web-based interfaces gave us a virtually limitless reservoir of data. The description and meaning of data is essential to channeling this overload. When we ask for information by searching indices we are really asking for data with certain kinds of meanings. If our search terms have different meanings in different databases, indices and searches will become flooded with numerous extraneous entries due to multiple uses of the same words.

In addition to our own need to understand the meaning of the data, we also must describe this meaning to collections of intelligent agents - to whom we now assign simple tasks, but upon whom we will rely increasingly to funnel the information tidal wave. 


\section{INFORMATION INTEROPERABILITY}

All too often a large amount of effort is needed to enable different databases to communicate with each other. In general there are two logical phases to achieving such information interoperability:

1) Data restructuring, and

2) Semantic transformations to ensure that the data is consistent and has the same meaning when different data items originate from different sources.

Even if two databases are both relational, it may be necessary to define data transformations through views or queries to combine data from multiple tables, transform data representations (e.g., date formats) and derive other data in order for the two databases to be logically compatible. When different databases have different data models - e.g., relational, objectoriented, or hierarchical - additional data restructuring is needed, usually involving a substantial amount of special purpose program code.

Nevertheless, compatibility at the data type and relation level is not enough, such simple things as units and precision must be compatible, and the more complex assumptions associated with the data must also be brought into coherence - for example does "employee" only refer to full time current employees or does it also include part-time consultants and retired employees?

The term metadata has been coined to refer to information about data, and this includes data type, schema information, data dictionary textual descriptions, and other semantic information. A formal study of how we use semantics to assign meanings to the raw data is the subject of ontology development, with important work taking place as part of applied AI (artificial intelligence).

In order for information interoperability to be effective on a large scale, we must have reusable tools that minimize the amount of effort and time needed to integrate each new information resource. We have had substantial success using a specification-based approach to data interoperability. We replace the process of writing specialized transformations with a concise high level description of a new data resource together with a (usually small) set of condition-action rules.

These specifications are processed to create an information mediator that carries out the necessary transformations when access is made to that data source. This is a particularly big win when non-relational data sources are involved as well as when relational data sources would otherwise require program directed non-trivial transformations.

The specification language can concisely describe very different data resources. These differences are captured in large part by annotations which govern the way the specification statements are applied. Finally, these specifications are compiled into an executable information bridge which mediates access to multiple data sources. The resulting combined data may be browsed uniformly through a common interface (Morgenstern 1995).

\subsection{Capturing the Meaning of Data}

A primary purpose of this paper is to provide a perspective on what is being done to capture the meaning of information - for which we will use the term metadata in its broadest sense. Metadata serves at least three purposes: (1) cataloging and then locating the relevant data when needed, (2) interpreting the form and format of the data, and (3) understanding the data: its meaning and semantics, and the context in which it is relevant and valid. Let us give some categories of metadata with examples of each:

- Data type: integer, float, double. gif-image

- Relational schema: table EMPLOY with attributes EMP-FN, EMP-LN, ID, .... 
- Schema constraints: each tuple has a different value for its key in a relational table, and the key is defined over the same attribute fields for each tuple.

- Object model: Employee is a subtype of Person.

- Semantic features units = celsius; averaged-over 1 minute; temperature taken at the surface.

- Derived feature: average age of group.

- Qualifying features: photo taken at 3pm 1/2/96, looking due east from center of intersection

- Constraints: age > 0; person-age < 200; person-age is subtype of age.

EMP.Salary < EMP.MGR.Salary

(i.e.: employee's salary must be less than manager's salary)

- Context: accuracy of $10^{-3}$ applies to micrographs number 20-23

- Standards: ID is Social Security Number; connector is type-5 pin

- Ontology: senior citizen; adolescent; disabled

In the last category above, the terms senior citizen, adolescent, and disabled do not generally have precise meanings (does a sprained ankle qualify one as disabled?). Thus the establishment of the particular meaning in a particular context may be more complex, and thus we refer to it as part of an ontology - a locally consistent vocabulary of defined terms and the interrelationship of these terms. These are only some of the categories by which one may organize the metadata, and these categories are not absolute.

In fact, some categories of metadata may describe and augment other metadata. For example, which attribute(s) form the key of a relational table is one form of metadata. A schema constraint such as referential integrity ${ }^{1}$ then is metadata which refers to and augments the metadata regarding the key values of the relation.

Metadata often is distinguished by the fact that a metadata definition applies to many data instances. So for example, there are potentially many tuples associated with the metadata which defines a relational table (Cercone 1990). This distinction blurs for derived, summary, and qualifying features, in which the feature's value may be associated with one collection of data, such as the average age of the equipment or the maximum elevation within a section of land.

The context of some information is a form of metadata which bounds the scope of applicability of this information. Context also provides a background in which this information is to be interpreted. Thus key information about a relational table has as its scope the tuples in that table. This key information also is to be interpreted in the context of the relational data model - in that it is not a pointer but rather a symbolic reference.

Context can be seen as an informal generalization of quantifiers from logic. Context all too frequently is unstated. We argue that it is important to make such metadata explicit as we want to share data from diverse data resources (Morgenstern 1996, 1989).

\subsection{The Vocabulary for Describing Semantics}

The terms and words we use to describe the metadata must have a meaning to us, and to our programs that use this information. This realization brings us to the work on ontologies, in which terminology is standardized and logically interrelated with other terminology in a consistent manner. An ontology is a representational vocabulary for the types of objects, concepts, and attributes, and the types of relationships which are important to represent.

For example, if we wish to describe spatial volume and shapes, we must also define three dimensions. If we wish to describe an engine, we should decide on the terms we are going to use - this is what the PDES/STEP ${ }^{2}$ product data description work is doing. If we are going to

1. Referential integrity requires that if there are existing references (e.g., from other tables) to this table, then the referenced key values must exist in this table. 
describe census data about poverty, we must agree on the meaning of our terms.

In effect, if we are going to succeed in describing the meaning of data, then the terms and words we use to express this description must already have an accepted meaning to us, and/or to our intelligent agent programs.

We focus on three areas below for metadata descriptions: 1) multimedia information, 2) document metadata, and 3) metadata that can help us to tame the World Wide Web.

\section{METADATA FOR MULTIMEDIA}

Insufficient attention has been given to capturing and utilizing metadata for multimedia information. The most basic metadata is its data type. This may be, for example, gif, tiff, bitmap, jpeg, mpeg, wav, or quicktime, among others. In the case of some formats such as quicktime, multiple media such as sound and video are coordinated and each medium may have its own particular type. All too often this data type information is indicated via an unenforced convention on the file name or implied in some other manner. Making such information explicit is a necessary first step.

Semantic features for multimedia are in their infancy and thus metadata for multimedia must be readily extensible, with new features being added as needed. Versioning may be needed if redefinition of features is prevalent. Metadata for multimedia typically will fall into two broad categories: externally obtained features, and derived features. We give examples of each.

Externally obtained features provide contextual and situational information such as the name of a picture, the date, time, and location of a video of a meeting, and perhaps a list of participants. Situational information may include the name of the author or 'photographer' who captured this information, the start and end points on the video tape and the actual elapsed clock time, as this may differ from the duration of the video itself due to breaks. Additional information may be needed for library filing, be it a traditional or digital library.

The second category of metadata involves features which can be derived from the multimedia itself. These features are perhaps more elusive as they depend on the current state of analysis techniques. Thus these features will evolve over time as technology becomes more sophisticated. Analysis may involve voice and speech recognition, optical character recognition (OCR), several forms of scene description and scene analysis - including face recognition, retrieval of similar scenes, morphing, isolation and tracking of moving figures in video, etc.

Derived features may include identification of each speaker through voice signatures and/ or images, tracking of each person in a video using motion analysis, and determining where in the video each person enters and exists the field of view. This would enable us to utilize such metadata to locate the segments of a video or audio tape where certain speakers are present.

Particular collections of metadata can be very useful for certain types of events, such as for a meeting or lecture. Such metadata may include points at which slides are changed during a meeting, identification of which person was speaking, and extraction of the text image from each slide. Optical character recognition (OCR) of this textual material can be used for indexing of such text images and also for indexing of the associated segments of the meeting video. This combination of metadata could provide a very useful summary of a long video. It also could be used to automatically create a table of contents with pointers into each segment of a long meeting or a series of lectures.

There are many other factors that may be worthy of extraction and representation as explicit metadata depending upon the application. The type of landscape or scenery may be useful, and for video the type of motion of the camera and of the participants may help to characterize

2. PDES stands for Product Data Exchange using STEP (STandard for the Exchange of Product model data). 
segments and even the whole film. When we say a film has a lot of 'action' we mean more than just rapid motion, but in fact rapid motion in many scenes is a good indicator of an 'action film.' Note that metadata features need not always be exact as we use these features to help us locate potentially relevant information in a much larger reservoir of stored data.

Some relevant features of audio include whether it is music, speech or noise, and its volume and tempo. Speech itself may be subjected to speech recognition to extract the words, and highlight keywords, in a similar manner to the use of OCR on text. More sophisticated techniques can enable automatic summarization of text extracted from slides and/or from speech. Automatic linking could be provided to related external data, documents, other multimedia resources.

In general, metadata is essential to effective prioritization and filtering of information, and this will become more important on a per user basis as people increasingly experience information overload. Furthermore it should be recognized that since data may have several uses, different metadata may be needed for each group of users (Morgenstern 1996).

We note that there is a growing body of work which provides retrieval of still photos based on similarity to other photos, including retrieval of similar faces. Given basic descriptors, there are some systems which will retrieve a prioritized set of images. Then the user can select those images that most closely approximate what the user is interested in - such as mountain scenes or similar faces - and the system will then retrieve and reprioritize a better set of matching scenes and photos. Within these systems, specially derived statistical features are extracted from each photo to facilitate the similarity retrieval. These features can be treated just like other derived metadata.

Furthermore, a system could opportunistically derive those features which are of lower priority during idle times for the system, thereby enabling a system to 'get to know' more about the multimedia it holds the longer that multimedia is in its repository. Also, as new techniques are developed and added to a system, these techniques can be opportunistically applied to older legacy data. While extensibility of metadata features will be useful in general, extensibility will be essential for multimedia information.

\section{DOCUMENT METADATA}

There is a wide range of metadata which may be important regarding documents, with different subsets being important for different applications. The structure of the document itself provides important metadata as to both the logical and the physical layout of the document's contents and the presentation of those contents to a reader. Such structure provides a handle on querying and rearranging the contents for alternative purposes.

The structure of the document is analogous to the schema description for a relational or object database. The Standard Generalized Markup Language (SGML) is increasingly the representation language of choice for document structure. Each document has a type which determines its general structure, as specified by the Document Type Definition (DTD). This DTD describes the components or building blocks which tailor the document's structure to a particular format, such as report, an article, or a book, etc. These components indicate, for example, which sections of text are paragraphs and how they are combined into particular sections and chapters, and that the latter two have headings but paragraphs do not have headings. The DTD and the composition of the components for a particular document constitute part of that document's metadata.

Metadata helps us to extract portions of a document for indexing and initial presentation. Only selected portions of a document such as the title and abstract might be used for indexing and as a preview of the document. As traditional book publishers look for ways to adapt to 
digital libraries, they already have found such metadata to be one dividing line between free services and pricing schemes. They are considering providing titles and abstracts freely, but charging for retrieval of the whole document. An outline of the article may be formed from the headings. Derived metadata such as automatic text summarization may augment document collections for the benefit of users.

The emergence of networked digital libraries are creating a flurry of activity among librarians. They now see an urgent need for more uniform standards regarding cataloging and indexing. We also now have the ability to keep track of more metadata attributes about each document, and it would be desirable to standardize many of these as well. Previously different physical libraries may have used somewhat different cataloging techniques (e.g. Dewey decimal versus Library of Congress numbers). It is now desirable to standardize on a common core of information that would enable digital libraries to collaborate and serve a much larger audience.

Below is a brief description of the 13 metadata elements that have been suggested for networked library resources and dubbed the Dublin Core Element Description (Weibel 1995):

Subject: The topic addressed by the work.

Title: The name of the object.

Author: The person(s) primarily responsible for the intellectual content of the object.

Publisher: The agent or agency responsible for making the object available.

OtherAgent: The person(s), such as editors and transcribers, who have made other significant intellectual contributions to the work.

Date: The date of publication.

ObjectType: The genre of the object, such as novel, poem, or dictionary.

Form: The data representation of the object, such as Postscript file or Windows executable file.

Identifier: String or number used to uniquely identify the object.

Relation: Relationship to other objects.

Source: Objects, either print or electronic, from which this object is derived, if applicable.

Language: Language of the intellectual content.

Coverage: The spatial locations and temporal durations characteristic of the object.

Of course, additional metadata information will be utilized in particular cases. In order to make these metadata categories clearer, below is an example of this Library Metadata description for one entry:

Title: On the Pulse of Morning

Author: Maya Angelou

Publisher: University of Virginia Library Electronic Text Center

OtherAgent: Transcribed by the University of Virginia Electronic Text Center

Date: 1993

Object: Poem

Form: 1 ASCII file

Source: Newspaper stories and oral performance of text at the presidential inauguration of Bill Clinton

\section{Language: English}

Note that while the particular categories are informative and useful, what is equally important is the potential for standardization on a particular set of metadata descriptors, as such 
standardization facilitates computerized access, categorization, and indexing.

While standardization is a desirable goal, one may question just how universal one standard can become. We suggest that one of the significant challenges for online data and document collections will be the technology to accommodate several alternative standards and categorizations. It is unlikely that a universal single categorization will be sufficient for all countries, languages, and disciplines. Moreover, autonomy may remain a priority in some cases.

Thus we recommend the notion of islands of consistent terminology, where an island is defined as a self-consistent collection of categories, terminology, and structure. When the implicit assumptions and meanings within an island are described explicitly, then one can build bridges between such islands of information. Furthermore, there will be multiple forms of overlapping descriptive information services that naturally arise, including literary bibliographies, cross-reference services, abstraction services, etc. Thus there will be further need to seamlessly utilize multiple data and metadata sources. We suggest that the information world of tomorrow will be more like an archipelago of interconnected islands of regionally selfconsistent information.

\section{METADATA FOR THE WEB}

The internet web is the fastest growing body of unstructured and semi-structured information. Increasingly structured formats are becoming available as the HTML standard is extended and as newer browsers incorporate more advanced features. HTML is the metadata language for current web pages, and its limitations are slowly becoming more obvious. Some support is now available for structuring of data into a table and for image maps which report the exact location of the user's mouse click; frames provide for multiple subwindows but otherwise provide little structure.

How to merge both highly structured data and unstructured information needs more careful attention. Consider a tabular representation. In some cases, one may wish each data value to be linked to information (metadata) such as source and accuracy. When using the data, we may wish to refer to individual data values, including the location of the data within the table and its supporting information, while at other times we may wish to refer to the whole table. For other purposes we may need to refer to a subset of rows and/or a subset of columns.

More generally we should be able to distinguish groups and subgroups of hypertext elements - we may refer to such a grouping as the scope of a reference. Currently, only the web page provides a scoping of information. While anchor points can be established within a page, such anchors currently are treated only as point locations rather than containers or region delimiters. Thus a subset of a page cannot be physically extracted without special purpose application code in java or a similar language.

Of equal importance is that a collection of web pages cannot be readily delimited and treated as a single collection. Thus if a large document is subdivided into a number of web pages for ease of access and traversal, the context of the whole document is lost. As a result we sometimes see a large document represented in two forms: 1) as a header page with link(s) to be followed to subsequent pages of reasonable size, and 2) as a single very large document which may be copied as a whole. These two forms are then separate, and changes to one are not reflected in changes to the other form. In fact, hypertext links tend to fragment the notion of a document as a reasonably self-contained and portable body of information.

Indexing of web-based information is in its early stages. It is now common to find from one thousand to several hundred thousand responses to a query against one index. These responses are segmented into small groups and may be presented in some approximate priority order. While a new query can be posed with more words as criteria for matching, the responses show 
that each word is used in many different ways, and the indexing scheme has no knowledge of different semantic uses of a word.

Sometimes words near the front of a document are treated with higher priority than words later in the document. At least one search engine uses the "Meta" tag of HTML to provide extra keywords and a very short description to be returned to the user. This does not really address the multiple semantics of many words. Furthermore, if we have a table of data values, there is no way to do selective searching on particular fields or columns, nor particular rows - all entries are treated uniformly as text. One might say that we need database retrieval techniques for such structured data. This would help. On the other hand, database queries do not conveniently address non-trivial text-based searches. For web indexing, a graceful combination of unstructured and structured querying should be supported - we are currently working on this.

\section{SUMMARY}

"It has been observed that distributed information systems, even though under control of a single administrative unit, create multiple problems. [These problems are] typically caused by differences in semantics and representation, [as well as] incomplete and incorrect data dictionaries (cataloging). These problems would be magnified manyfold in any distributed information system...." (Desai 1995). Our challenge is to describe diverse information resources in ways which are locally consistent as well as relatable to other information collections which may use different representations, semantics, and assumptions. We need to provide a combination of free form and structured queries, not only to retrieve separate web pages, but also to create tailored collections of pages with intelligent browsing capabilities.

\section{REFERENCES}

Cercone, N., Morgenstern, M, Sheth A., and Litwin W. (1990) Resolving Semantic Heterogeneity, Sixth Int'l Conference on Data Engineering, Los Angeles.

Desai, B. (1995) The Semantic Header and Indexing and Searching on the Internet, Concordia University, URL: http://www.cs.concordia.ca/ faculty/bcdesai/cindi-system-1.1.html

Morgenstern. M. (1996), Active Meta-Data Dictionary to Support Heterogeneous Databases, Xerox Design Research Institute, DARPA report.

Morgenstern. M. (1995) A Database Integration Platform for Concurrent Engineering, Xerox Design Research Institute, ARPA report.

Morgenstern, M. (1989) Constraint-Based Systems: Knowledge About Data, Expert Database Systems, Benjamin Cummings/Addison Wesley.

Weibel, S., Godby, J., Miller, E. and Daniel, R. (1995) OCLC/NCSA Metadata Workshop Report, Office of Research, OCLC Online Computer Library Center, Inc., also known as The Dublin-Core Metadata Report, URL: http://www.oclc.org:5046/conferences/metadata/ dublin_core_report.html

\section{BIOGRAPHICAL INFORMATION}

Matthew Morgenstern is a Principal Scientist with Xerox's Design Research Institute and is a Visiting Fellow with the Department of Computer Science at Cornell University. His doctorate in Computer Science is from the Massachusetts Institute of Technology. He is the principal investigator for projects on heterogeneous database integration and on active metadata management for distributed databases. He also has been active in computer security and has developed a model for multi-level security of object-oriented databases. 